\title{
Assistência circulatória mecânica: porque e quando
}

\section{Mechanical circulatory support: why and when}

\author{
Alfredo Inácio Fiorelli', José de Lima Oliveira Junior², Guilherme \\ Henrique Bianchi Coelho ${ }^{3}$, Diogo Cristo da Rocha ${ }^{4}$
}

Fiorelli Al, Oliveira Junior JL, Coelho GHB, Rocha DC. Assistência circulatória mecânica: porque e quando. Rev Med (São Paulo). 2008 jan.-mar.;87(1):1-15.

\begin{abstract}
RESUMO: A insuficiência cardíaca tem avançado ao longo dos últimos anos tendo em vista o envelhecimento da população, o maior índice de sobrevida após infarto agudo do miocárdio pelo uso dos trombolíticos, o avanço nas operações cardíacas e a melhora no diagnóstico das cardiomiopatias. A doença não tem cura e o seu curso natural é inevitável, a despeito das diferentes tentativas de mudá-la, e o máximo que se consegue é retardar o seu desfecho. O transplante cardíaco representa a única forma existente na atualidade capaz de mudar drasticamente esse panorama por oferecer melhora significativa na qualidade de vida e na sobrevida em longo prazo. Todavia o procedimento é limitado a poucos, principalmente pela falta de doadores de órgãos, e muitos morrem na fila aguardando a operação. A assistência circulatória mecânica surge como uma forma de suporte artificial para permitir que muitos possam chegar até o transplante, funcionado como uma verdadeira ponte ao procedimento. Nos casos agudos de insuficiência cardíaca, nas quais o miocárdio apresenta condições de recuperação, a assistência mecânica permite oferecer condições hemodinâmicas favoráveis para que o paciente não desenvolva falência de múltiplos órgãos e o miocárdio em repouso relativo tenha condições de recuperação. Esta última modalidade é denominada de ponte de recuperação. Mais recentemente discute-se o uso dos ventrículos e dos corações artificiais como terapia de destino como implante definitivo naqueles pacientes com contra-indicação ao transplante ou que recusam o procedimento. O presente trabalho tem por finalidade apresentar uma ampla revisão sobre o lugar que a assistência circulatória mecânica ocupa como método auxiliar no tratamento da insuficiência cardíaca.
\end{abstract}

DESCRITORES: Insuficiência cardíaca congestiva/patologia. Circulação assistida/métodos. Transplante de coração/tendências. Fatores de risco. Qualidade de vida.

1. Diretor da Unidade de Perfusão e Assistência Cardiorrespiratória/Transplante Cardíaco do InCor-HCFMUSP.

2. Pós-graduando do Departamento de Cardio-Pneumologia da FMUSP e Aluno do Curso de Especialização em Transplante Cardíaco - InCor-HCFMUSP.

3. Coordenador da Liga de Transplante Cardíaco da FMUSP e aluno do Curso de Especialização em Transplante Cardíaco - InCor-HCFMUSP.

4. Acadêmico da FMUSP e Membro da Liga de Transplante da FMUSP.

Endereço para correspondência: fiorelliai@ uol.com.br 


\section{INTRODUÇÃO}

A insuficiência cardíaca é um problema de saúde pública mundial, sendo a maior causa de mortes em diversos países e é considerada epidêmica pelo The National Institute of Health dos Estados Unidos da América ${ }^{1}$. A constante evolução das estratégias terapêuticas para as doenças ateroscleróticas coronárias, valvares, congênitas, entre outras, permitiu que a sobrevida destes pacientes aumentasse significativamente nas últimas décadas ${ }^{2}$.

Com o passar do tempo, ocorrem uma série de alterações moleculares, bioquímicas, celulares e estruturais no miocárdio, denominadas recentemente de remodelamento ventricular ${ }^{3-8}$, que podem ser reversíveis, total ou parcialmente ${ }^{5,9}$. Estudos recentes sugerem que a utilização de dispositivos de assistência circulatória mecânica, nestes pacientes, pode auxiliar na reversão das alterações estruturais causadas no miocárdio, denominadas de remodelamento reverso ${ }^{10}$, e, conseqüentemente, com melhora funcional ${ }^{11-13}$.

Estima-se que somente nos Estados Unidos surgem cerca de 500.000 novos pacientes com falência cardíaca por ano ${ }^{14}$ e com os avanços terapêuticos observa-se o prolongamento da sobrevida destes pacientes e, conseqüentemente, acarreta elevação da incidência de insuficiência cardíaca com impacto direto social e econômico para o sistema de saúde.

O tratamento da falência cardíaca é visto, hoje em dia, como uma subespecialidade médica dentro da cardiologia, constituindo-se numa grande fronteira tanto para a cardiologia quanto para a cirurgia cardiovascular. A criação de centros especializados no atendimento dos pacientes com insuficiência cardíaca tem se expandido em diferentes paises, inclusive em nosso meio, permitindo melhor atendimento aos pacientes e a otimização da terapêutica. As denominadas Unidades de Tratamento da Insuficiência Cardíaca são constituídas por uma equipe multiprofissional que congregam diferentes especialistas, tais como: médicos especializados no tema, enfermeiros, nutricionistas, psicólogos e assistentes sociais.

A despeito da enorme evolução verificada na terapia medicamentosa com a introdução do uso de inibidores da enzima de conversão da reninaangiotensina (inibidores da ECA), beta-bloqueadores e outros, foi possível modificar significativamente a evolução natural da doença. Todavia, isto não significa a sua cura, pois o seu curso é inevitável. A insuficiência cardíaca em fase avançada mata mais do que o infarto agudo do miocárdio e muitas modalidades de câncer como o de mama, bexiga e próstata, perdendo apenas para o de pulmão. Atualmente, a única forma capaz de interromper o curso da doença e oferecer retorno às condições hemodinâmicas normais é o transplante cardíaco. Todavia, esta modalidade não é isenta de riscos e efeitos colaterais. O procedimento não é de fácil execução e não pode ser oferecido a todos, principalmente pela carência de doadores de órgãos.

Anualmente são realizados cerca de 2500 a 3000 transplantes cardíacos nos Estados Unidos ${ }^{1}$, o que do ponto de vista epidemiológico não tem grande impacto, tendo-se em vista o número de pacientes que potencialmente se beneficiariam com o método. Desta forma, fica clara a necessidade da adoção de alternativas terapêuticas para suportar pacientes que estão em lista de espera para o transplante e apresentam piora clínica, ou para aqueles que são contra-indicados por razões diversas. Nestas situações especiais o uso de dispositivos capazes de manter as condições hemodinâmicas dos pacientes por período prolongado e substituir, total ou parcialmente, de forma temporária ou definitiva, as funções de bomba do coração apresentam lugar especial. Ao conjunto de técnicas e equipamentos capazes de realizar essas façanhas denomina-se de assistência circulatória mecânica, podendo receber outras denominações, tais como: suporte circulatório, suporte mecânico, circulação assistida e outros.

Os diferentes tipos de dispositivos e as táticas de assistência circulatória permitem que o método possa ser utilizado nas afecções crônicas e agudas, bem como em miocardiopatias de origens diversas. Como não existe um equipamento ideal, sempre que possível procura-se conciliar dentro do arsenal existente aquele que for o mais adequado e disponível. Quando a assistência é utilizada como suporte temporário no miocárdio viável até que ele se recupere é denominada de "terapia ou ponte de resgate", e nas situações onde o miocárdio é considerado irrecuperável e o paciente está em fila de espera aguardando transplante denomina-se de "ponte para o transplante". Mais recentemente tem se discutido de forma ampla a utilização de substitutos ventriculares permanentes, quando o paciente recusa o transplante ou apresenta alguma contra-indicação, caracterizando a "terapia de destino".

Inicialmente os dispositivos de assistência circulatória mecânica foram liberados pelo Food and Drugs Administration apenas para uso por curto período de tempo, como ponte para transplante, por aumentar a perfusão tecidual em diversos órgãos, a capacidade funcional dos pacientes ${ }^{15-18}$, e os índices de qualidade de vida. Diferentes estudos sugerem, ainda, que estes dispositivos podem reverter algumas alterações hemodinâmicas ${ }^{19-22}$ e marcadores moleculares de disfunção miocárdica ${ }^{4,23}$, após 
manter o coração em repouso relativo por período diverso. Caso não seja possível a recuperação do coração lesado, os dispositivos apresentam outra função primordial: a de impedir que o baixo débito leve a alterações irreversíveis do organismo, o que fatalmente culminaria com a morte do paciente.

Com o desenvolvimento crescente, principalmente na última década, dos dispositivos de assistência circulatória e com a limitação do número de doadores, estes dispositivos passaram a ser utilizados mais precocemente. Gradativamente essa atividade estendeu-se a um maior número de pacientes, e houve também a ampliação do período de implante, permitindo cada vez mais o emprego nas diferentes formas de terapia: de recuperação, ponte para o transplante ou de destino ${ }^{25}$.

Os primeiros passos na assistência circulatória mecânica foram dados por Le Gallois, em 1812 ao postular que o coração poderia ser substituído por uma máquina, desde que esta fosse capaz de manter fluxo sangüíneo adequado para suprir as necessidades periféricas.

De Bakey, em 1934,14,25, deu o passo decisivo no desenvolvimento da assistência circulatória mecânica com a idealização da bomba de propulsão sangüínea com roletes, que somente em 1953 foi incorporada à circulação extracorpórea. Em 1957, Stukey empregou com sucesso uma máquina de circulação extracorpórea, como suporte circulatório, em um paciente em choque cardiogênico, pós-infarto agudo do miocárdio.

$\mathrm{Na}$ década de 1970, as buscas pelo desenvolvimento de dispositivos mais efetivos de assistência circulatória criaram basicamente duas linhas de pesquisa. Uma voltada para a construção de um coração artificial total, que substituísse integralmente o coração nativo e servisse de suporte até o transplante. A outra seguiu a linha do desenvolvimento de um suporte ventricular mecânico, uni ou bicameral, e que oferecesse assistência até a recuperação do coração ou mantivesse as condições hemodinâmicas do paciente até a realização do transplante ${ }^{26}$.

Diferentes pesquisas vêm sendo realizadas ao longo dos anos para tentar vencer grandes obstáculos impostos por esta nova modalidade terapêutica e chegar o mais próximo do que se considera o dispositivo ideal, onde vêm se destacando as seguintes características: a durabilidade do equipamento, facilidade no implante, ausência de defeitos mecânicos, possibilidade de utilização em pacientes com diferentes pesos corpóreos, menor interação com os elementos sangüíneos e baixo custo, entre outros.

Para atender aos pacientes em fila de transplante cardíaco que venham requerer os benefícios da assistência circulatória têm-se desenvolvido dispositivos que suportem funcionamento por longos períodos até que se consiga um doador adequado. Durante esse período os dispositivos devem oferecer fluxo sanguíneo adequado com o menor grau de lesão aos elementos figurados do sangue, bem como menor índice de ativação dos diferentes sistemas de cascata, como o da cascata da coagulação, e permitir a deambulação do paciente.

Mais recentemente tem-se retomado investigações para a utilização da assistência circulatória como terapia de destino para aqueles pacientes que não podem se beneficiar do transplante por diferentes razões. Essa nova geração pode oferecer assistência circulatória total, coração artificial, ou ao ventrículo esquerdo, ventrículo artificial, e resultados recentes têm demonstrado vantagens superiores ao tratamento clínico exclusivamente.

Assim, a assistência mecânica atualmente pode ser indicada nos casos de disfunção miocárdica aguda, como no choque cardiogênico pós-cardiotomia, indicação mais comum atualmente, nas miocardites infecciosas ou tóxicas, funcionando como suporte temporário até o restabelecimento da função ventricular ou ponte para transplante.

A utilização de dispositivos de assistência circulatória mecânica por tempo prolongado teve sua eficácia comprovada em relação ao tratamento clínico num grande estudo prospectivo e aleatório, denominado Rematch ${ }^{23}$. Desta forma, pacientes que apresentavam contra-indicação ou recusaram o transplante foram distribuídos de forma aleatória e acompanhados prospectivamente, e se pode observar melhor evolução nos pacientes que receberam assistência como terapia definitiva em relação aqueles com tratamento clínico exclusivamente.

A utilização prolongada da assistência circulatória mecânica comprovadamente é capaz de reverter diferentes alterações celulares observadas na fase avançada da falência miocárdica. Podese observar reversão da redução da atividade adrenérgica, da expressão de citocinas inflamatórias pelo miocárdio $22,27,28$, entre outras, e essas modificações celulares são acompanhadas de substancial melhora clínica do paciente. Nota-se redução da hipertensão pulmonar e melhora da função renal ${ }^{29,30}$, fazendo com que alguns pacientes possam ter as suas contra-indicações para o transplante revertidas e ingressarem na fila de espera, como demonstrado por Young et al. ${ }^{30}$.

Da mesma forma, estes mesmos autores observaram que alguns pacientes com assistência circulatória implantada inicialmente como ponte para transplante experimentam melhora significativa 
da função ventricular, a ponto de se sustentar sem nenhum outro dispositivo. Em outros casos, em virtude de complicações neurológicas, os pacientes passam a ter uma contra-indicação formal ao transplante. Essa difícil decisão deveria ser baseada em avaliação individual dos riscos e benefícios das diferentes formas de terapia, levando-se em consideração que a ocorrência de certas complicações inerentes à assistência são imprevisíveis.

Nas indicações da assistência, principalmente as de destino, devem-se levar em consideração os elevados custos na utilização da terapia e a sua utilização deve ser orientada também em função da possível melhora clínica e não exclusivamente nos critérios de elegibilidade. Para pacientes com deterioração aguda da função miocárdica, como pós-cardiotomia, choque cardiogênico oriundo de infarto agudo do miocárdio ou miocardite fulminante, os dispositivos de suporte de curto prazo são melhor opção terapêutica, seja como ponte para recuperação funcional do ventrículo, como ponte para transplante ou até como ponte para dispositivos de destino. Esta última modalidade é também denominada de ponte para ponte.

Nos pacientes portadores de insuficiência cardíaca crônica a assistência circulatória de longo prazo deve ser feita com dispositivos que ofereçam suporte como ponte para o transplante ou para terapia destino.

\section{ASPECTOS FISIOLÓGICOS}

\section{Fisiologia ventricular normal}

O sistema cardiovascular pode ser visto, do ponto de vista hidrodinâmico, como duas bombas que operam em série, cada uma delas com pós-carga específica, determinada pela resistência vascular sistêmica ou pulmonar. Na Figura 1 encontrase esquematizada as possíveis interações dos dispositivos de assistência circulatória mecânica com os ventrículos e suas respectivas pós-cargas.

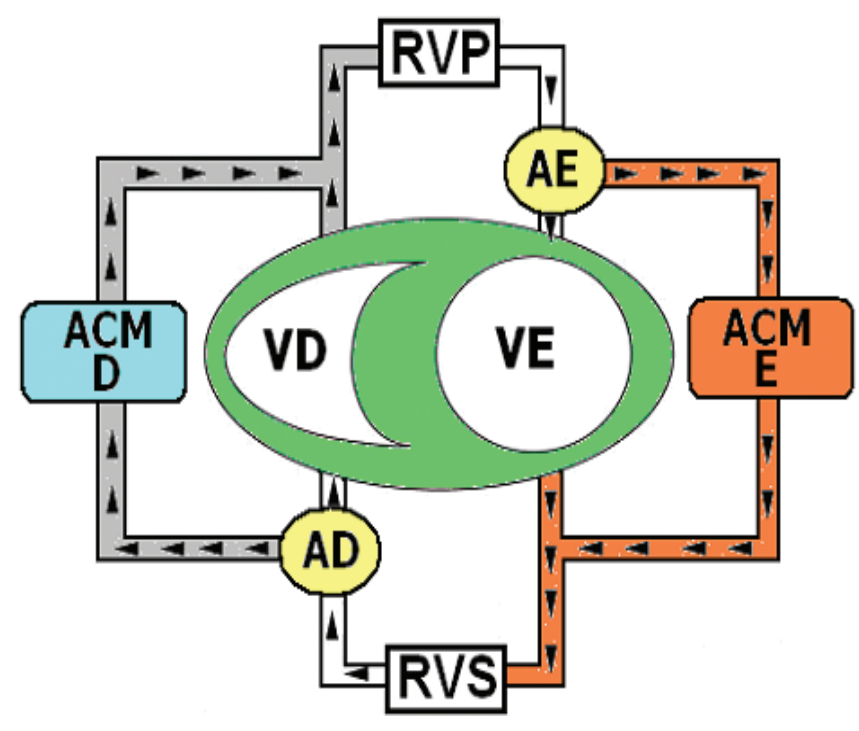

Figura 1. Esquema da relação entre os ventrículos direito(VD) e esquerdo(VE) e as diferentes modos de assistência circulatória direita(ACM-D) e esquerda(ACM-E). A ACM-D auxilia o VD a vencer a resistência vascular pulmonar(RVP) e a ACM-E auxilia o VE a resistência vascular sistêmica(RVS). Em ambas as situações há desvio do sangue dos ventrículos, colocando-os em repouso relativo. Na ACM-D o sangue pode ser derivado do átrio direito(AD) ou diretamente do VD e lançado no tronco pulmonar. Na ACM-E o sangue pode ser drenado do átrio esquerdo(AE) ou diretamente do VE e injetado diretamente na aorta ou em uma das artérias femorais

Em condições normais cada câmara ventricular apresenta correlação direta entre o volume diastólico final e a pressão diastólica final, onde diferentes fatores interferem diretamente mutuamente entre si, destacando-se a contratilidade de cada ventrículo e as condições de pré e pós-carga. A interação entre elas determina o comportamento da curva de pressão e volume ventricular, tendo como fatores determinantes:

- o retorno venoso, considerado como pré- 
carga, está associado ao volume sistólico final e ao volume diastólico final;

- a pré-carga e a contratilidade ventricular que interferem no volume diastólico final;

- o volume sistólico é afetado pela pós-carga e pelo estado contrátil do ventrículo;

- a pressão ventricular sistólica, depende do volume sangüíneo ejetado e da resistência vascular imposta a cada ventrículo.

\section{Fisiopatologia da insuficiência ventricular}

Nas disfunções ventriculares avançadas, agudas ou crônicas, independentemente da etiologia da afecção, o coração é incapaz de bombear sangue de forma eficaz para suprir a demanda tecidual sistêmica, ou só a faz às custas de pressão de enchimento muito elevado, sendo incompatível com a sobrevida do paciente em curto espaço de tempo. Considerando-se que o coração apresenta duas bombas ventriculares é de fundamental importância a distinção se a falência é de origem esquerda, direita ou ambas. Na prática clínica, em geral, existe o comprometimento biventricular, com predomínio de uma delas. Ainda mais, o comprometimento de um dos ventrículos em geral determina em tempo variável a disfunção da câmara contra-lateral. $\mathrm{Na}$ alta prevalência do infarto agudo do miocárdio, nas miocardites e nas disfunções ventriculares após cardiotomia, entres outras causas, o ventrículo esquerdo é o mais comprometido e responde pela maior incidência de insuficiência ventricular.

Deve-se destacar ainda a importância no diagnóstico da lesão ventricular se o comprometimento acarreta prejuízo da função sistólica, diastólica ou ambas, o que é o mais freqüente. As disfunções isoladas existem e caracterizam diferentes entidades clínicas. Todavia, a sua concomitância é o padrão habitual, com predomínio de uma delas.

A disfunção sistólica determina redução da fração de ejeção e aumento do volume diastólico final, desencadeando um processo de remodelamento ventricular, ou seja, alteração da geometria do ventrículo na tentativa de se adaptar as novas condições hemodinâmicas. O remodelamento ventricular deve ser entendido como prejudicial à função do ventrículo, onde a velocidade e a intensidade do remodelamento dependem, entre outros fatores, do tipo e do grau da lesão ao coração. O débito cardíaco pode não sofrer sérias alterações na fase inicial do processo, porém, com o passar do tempo e a persistência do processo lesivo ao miocárdio, há tendência à sua redução gradativa. Este fenômeno é responsável pela deflagração de uma série de mecanismos compensatórios, tais como: ativação sistema renina angiotensina aldosterona, liberação do hormônio antidiurético e elevação do nível de catecolaminas circulantes, com conseqüente aumento da pós-carga e da volemia.

Fisiologia do restabelecimento hemodinâmico pela assistência circulatória mecânica

Os dispositivos de assistência circulatória mecânica têm a função de restabelecer o débito cardíaco efetivo total ou parcialmente, garantir a perfusão sistêmica e evitar a disfunção de múltiplos órgãos. Com a restauração do débito cardíaco há aumento da pressão arterial e redução das pressões em câmaras esquerdas. Conseqüentemente, ocorre melhora da congestão pulmonar, redução da pressão venosa central e diminuição da atividade neurohormonal ativada pela insuficiência cardíaca. Todavia, se houver retardo na indicação da assistência circulatória ou o paciente já estiver com a falência de múltiplos órgãos em curso, o óbito será quase que inevitável.

Principalmente nos casos de insuficiência cardíaca de recente início, como anteriormente citado, encontra-se o perfil de pacientes onde a assistência circulatória mecânica é capaz de oferecer condições para que o miocárdio possa restaurar o seu estado funcional ${ }^{31,32}$. Nos casos agudos é mais freqüente o encontro de miocárdio lesado, porém, com possibilidade de recuperação desde que tenha condições para tal. Ao contrário, esta mesma situação é menos freqüente nos casos de longa evolução de insuficiência cardíaca pelo predomínio da substituição do miocárdio normal por fibrose. Nesta última situação a assistência circulatória é utilizada como ponte para o transplante.

Há evidências na literatura de que reduções na tensão da parede ventricular, com uso de dispositivos de assistência esquerda por períodos prolongados, altera a expressão gênica anormal verificada nos miócitos de pacientes com falência ventricular, há redução da hipertrofia celular, do depósito de colágeno e de fibrose intersticial ${ }^{33,34}$. Pode-se observar ainda redução da miocitólise, da liberação miocárdica de mediadores inflamatórios (IL6, IL8 e TNF), da liberação sistêmica de catecolaminas, hormônio natriurético atrial e de vasopressina ${ }^{35}$.

A despeito do crescente número de evidências do impacto funcional e anatômico sobre o ventrículo, a utilização de dispositivos de assistência circulatória mecânica por períodos prolongados ainda não está completamente estabelecida, sendo necessário maiores estudos e a criação de protocolos específicos. 


\section{Interação fisiológica entre os dispositivos e o coração nativo}

\section{Sincronização com o coração nativo}

A maioria dos dispositivos de assistência circulatória não está em sincronismo com o ciclo cardíaco, o que é chamado de "em fase", ou seja: a sístole ventricular ocorre durante a diástole do dispositivo, determinando máximo enchimento deste e redução da pressão de enchimento do ventrículo esquerdo do paciente. Quando o dispositivo é programado "fora de fase", coração e diapositivo têm sístoles coincidentes, o que gera uma competição pelo fluxo sanguíneo, reduzindo o enchimento do dispositivo e elevando as pressões ventriculares (Figura 2).

\section{INDICAÇÕES DE ASSISTÊNCIA CIRCULATÓRIA MECÂNICA}

\section{Critérios clínicos}

Os principais critérios utilizados na seleção dos pacientes à assistência circulatória mecânica encontram-se discriminados na Tabela 1.

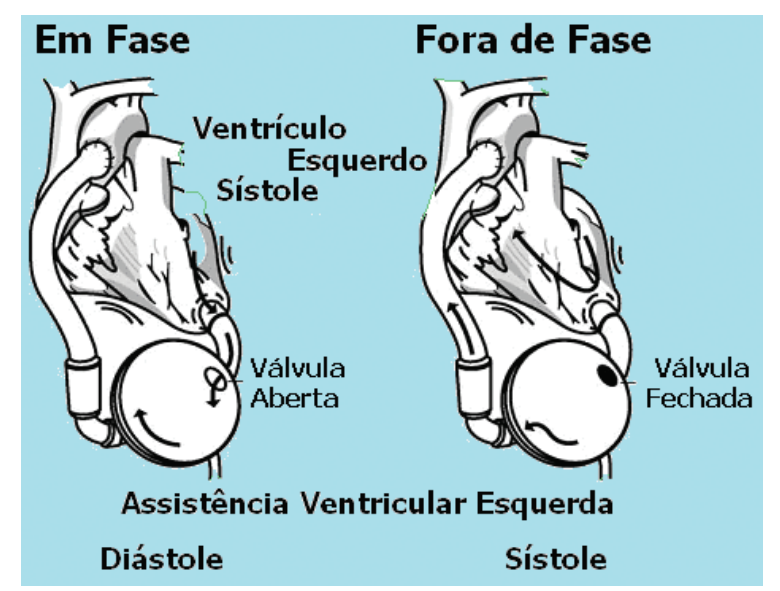

Figura 2. Dispositivo de assistência ventricular esquerda

Tabela 1. Critérios utilizados na seleção dos candidatos à assistência circulatória mecânica

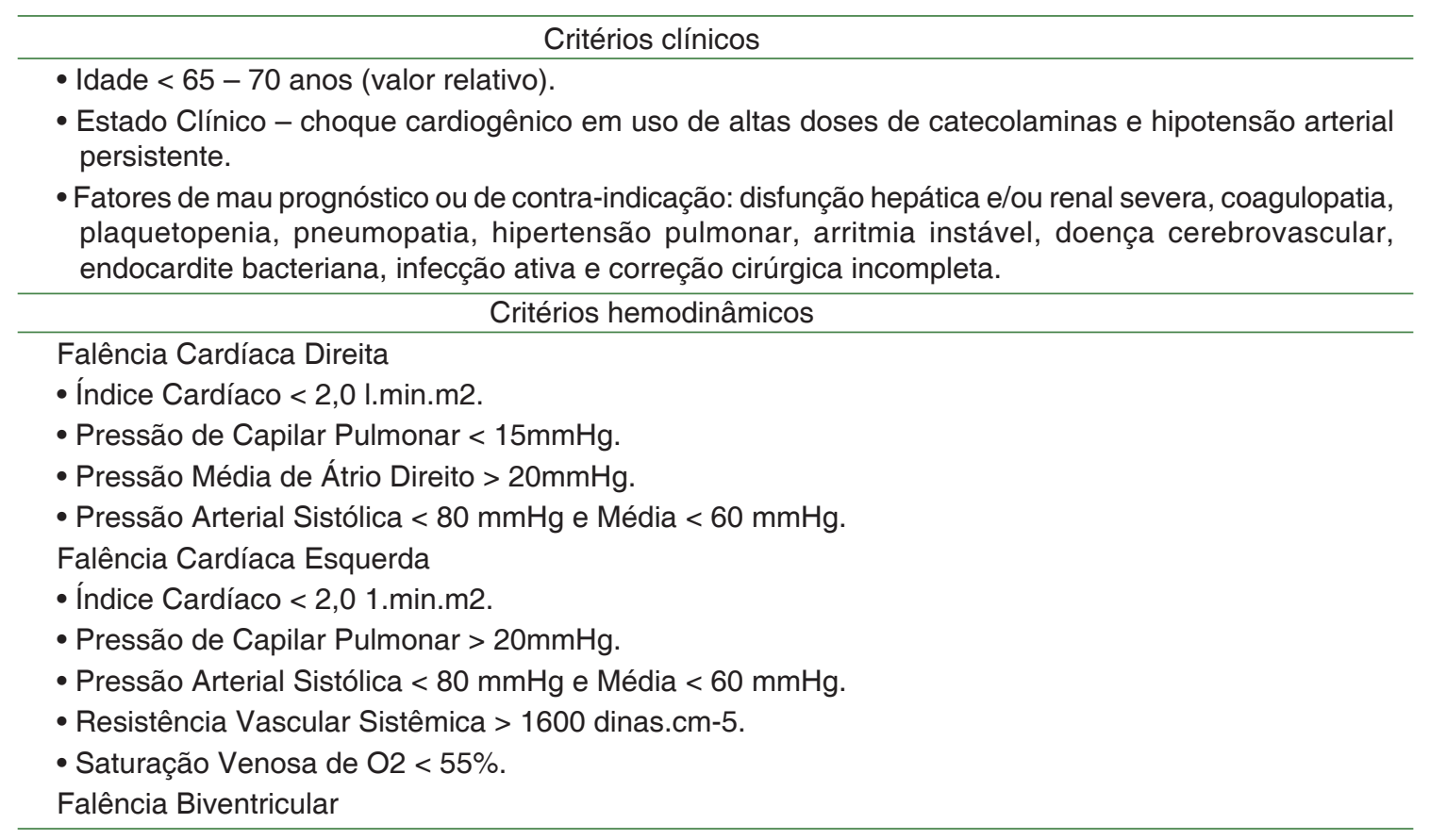




\section{CLASSIFICAÇÃO DOS DISPOSITIVOS DE ASSISTÊNCIA CIRCULATÓRIA MECÂNICA}

Os dispositivos de assistência circulatória mecânica podem ser classificados em função de diferentes critérios, como apresentado na Tabela 2.

Tabela 2. Classificação dos dispositivos de assistência circulatória mecânica

\begin{tabular}{|c|c|c|}
\hline \multicolumn{3}{|l|}{ Tipo de Fluxo } \\
\hline - Contrapulsação & \multirow{3}{*}{\multicolumn{2}{|c|}{$\begin{array}{l}\text { Balão intra-aórtico. } \\
\text { Roletes, centrífuga e axial. } \\
\text { Pneumático e elétrico. }\end{array}$}} \\
\hline - Contínuo & & \\
\hline • Pulsátil & & \\
\hline Posição em relação ao coração & \multicolumn{2}{|c|}{ Série ou Paralelo. } \\
\hline Em relação ao ventrículo assistido & \multicolumn{2}{|c|}{ Direito, esquerdo ou biventricular. } \\
\hline Grau de substituição ventricular & \multicolumn{2}{|c|}{ Total ou parcial. } \\
\hline Posição em relação ao paciente & \multicolumn{2}{|c|}{ Pára-corpóreo ou implantável. } \\
\hline Tempo de permanência & $\begin{array}{l}<30 d \\
\text { 30d. - 1ano } \\
>1 \text { ano }\end{array}$ & $\begin{array}{l}\text { curta duração } \\
\text { média duração } \\
\text { longa duração }\end{array}$ \\
\hline
\end{tabular}

Os dispositivos para curta duração são empregados na maior parte das vezes em pacientes com choque cardiogênico após infarto agudo do miocárdio, após cirurgia cardíaca ou em casos de miocardites, em geral como suporte para recuperação da função ventricular, ponte para um dispositivo de longa duração ou para transplante.

Os dispositivos de média duração são empregados naqueles pacientes com cardiomiopatia que evoluem em baixo débito e geralmente servem como ponte para transplante. Os dispositivos de longa permanência são de indicação mais controversa, sendo utilizados como terapia de destino em pacientes com contra-indicação ou recusam o transplante. $\mathrm{Na}$ escolha do tipo de assistência circulatória mecânica e do dispositivo mais adequado, deve-se levar em consideração a etiologia da disfunção ventricular, o tempo previsto de assistência e as condições clínicas momentâneas do paciente.

Os dados coletados pelo Registro Internacional da International Society for Heart and Lung Transplantation até $2004^{36}$ mostram a distribuição dos diferentes modos de suporte circulatório mecânico em função da câmara ventricular assistida na Tabela 3 e das suas principais indicações na Tabela 4.

Tabela 3. Distribuição dos dispositivos em função da câmara ventricular assistida*

\begin{tabular}{|c|c|c|}
\hline Assistência ao Ventrículo Esquerdo & 339 & \\
\hline Fluxo Pulsátil - Dispositivo Crônico & & 303 \\
\hline Fluxo Contínuo - Dispositivo Crônico & & 27 \\
\hline Não especificado & & 9 \\
\hline Assistência Biventricular & 74 & 69 \\
\hline VE: Fluxo Pulsátil - Dispositivo Crônico & & 1 \\
\hline VE: Fluxo Contínuo - Dispositivo Crônico & & 4 \\
\hline \multicolumn{3}{|l|}{ VE: Não especificado } \\
\hline VD: Fluxo Pulsátil - Dispositivo Crônico & & 44 \\
\hline VD: Fluxo Pulsátil - Dispositivo Temporário & & 21 \\
\hline VD: Fluxo Contínuo - Dispositivo Temporário & & 8 \\
\hline VD: Não especificado & & 1 \\
\hline Total & 413 & \\
\hline
\end{tabular}

\footnotetext{
* Registro Internacional da ISHLT - 2004
} 
Fiorelli AI et al. Assistência circulatória mecânica: porque e quando.

Tabela 4. Indicações de uso de assistência circulatória mecânica*

\begin{tabular}{lll}
\hline Indicação & $\mathrm{N}^{\circ}$ & $\%$ \\
\hline Ponte para o Transplante & 312 & $75,5 \%$ \\
Ponte para Recuperação & 24 & $5,8 \%$ \\
Terapia de Destino - Idade Avançada & 19 & $4,6 \%$ \\
Terapia de Destino - Morbidades Associadas & 12 & $2,9 \%$ \\
Terapia de Destino - Hipertensão Pulmonar fixa & 3 & $0,7 \%$ \\
Terapia de Destino - Contra-indicação para o Tx & 1 & $0,2 \%$ \\
Não especificada & 7 & $1,7 \%$ \\
\hline
\end{tabular}

* Registro Internacional da ISHLT - 2004

\section{Tipos de dispositivos}

\section{Balão intra-aórtico}

Este método de assistência circulatória utiliza o princípio de contrapulsação idealizado inicialmente por Harken, em 195837. Contudo, deve-se a Moulopoulos et al ${ }^{38}$, em 1962, a descrição dos benefícios da aplicação de um cateter-balão introduzido na aorta descendente de cães e acionado sincronicamente em regime de contrapulsação.

Após diferentes aprimoramentos, Kantrowitz et al., em $1968^{39}$, relataram a primeira aplicação clínica deste método. O balão intra-aórtico é um sistema se assistência circulatória mecânica de simples instalação, de eficiência comprovada e seguro, de tal forma que se transformou na primeira opção de suporte circulatório no tratamento de falência ventricular esquerda refratária à terapêutica convencional.

O balão intra-aórtico é composto basicamente de um console e de um cateter-balão, sendo acoplado em série com o coração. O cateter é flexível e apresenta a extremidade distal radiopaca para facilitar a identificação do seu correto posicionamento próximo à emergência da artéria subclávia esquerda.

O balão é insuflado com gás hélio, que é inerte, volátil, com baixa viciosidade e permite acionamento eficiente mesmo em freqüências elevadas. Atualmente os acidentes com extravasamento de gás são raros devido à qualidade do material empregado na construção do cateter. O acesso preferencial para introdução do cateter-balão é a via femoral. Todavia, outras artérias podem ser utilizadas em situações especiais, tais como: ilíacas, subclávia esquerda e aorta ascendente.

Após a introdução do cateter é possível se obter o registro da curva de pressão na aorta ascendente, que servirá como um dos sinais para o acionamento do balão intra-aórtico. A aorta ascendente pode ser utilizada, em cirurgia cardíaca, como via alternativa para introdução do cateter-balão quando o acesso femoral não for possível. O cateter é posicionado na aorta descendente no mesmo local que pela via femoral, porém com progressão anterógrada.

O acesso pelas artérias subclávias ou ilíacas pode ser feito em situações excepcionais. Cochran et al., em 2002, propôs o acesso pela artéria subclávia para uso prolongado como ponte para o transplante, permitindo inclusive o acompanhamento ambulatorial dos pacientes. O console contém o sistema elétrico e mecânico que controla o acionamento do cateterbalão.

Os ajustes adequados de insuflação e desinsuflação sincrônica do balão podem ser feitos com o ritmo cardíaco do paciente, e o disparo deflagrado pelo sinal do eletrocardiograma ou pela curva depressão arterial. O sinal obtido da curva de pressão pela monitorização na aorta ascendente é mais sensível do que em artéria periférica.

A deflagração do balão intra-aórtico pelo sinal eletrocardiográfico é programada para iniciar a insuflação no meio da onda $\mathrm{T}$, quando tem inicio a diástole ventricular, e a desinsuflação antes do inicio do próximo complexo QRS, momento em que se inicia a sístole ventricular. Na deflagração acionada pela curva de pressão arterial, ajusta-se a insuflação do balão no fechamento da valva aórtica, determinada na curva pela incisura dicrótica.

A insuflação precoce, com a valva aórtica ainda aberta, acarreta refluxo de sangue ao ventrículo e aumenta indesejavelmente o trabalho do coração (Figura 3). Por outro lado, a insuflação tardia reduz a elevação desejada da pressão diastólica na aorta, diminuindo, portanto, o seu rendimento. 


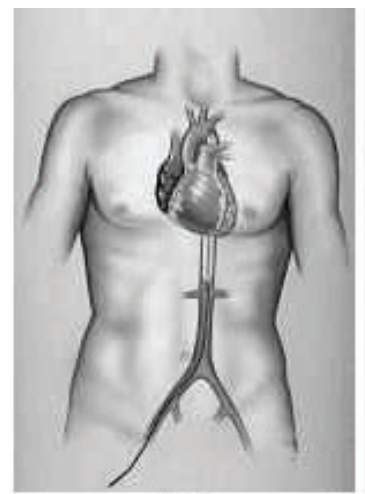

1A

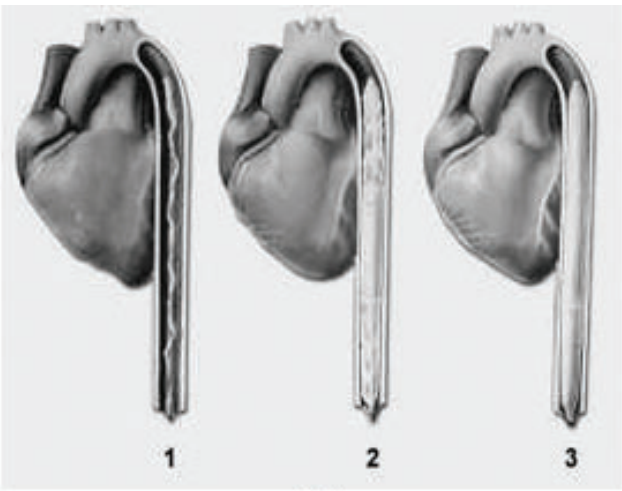

1B

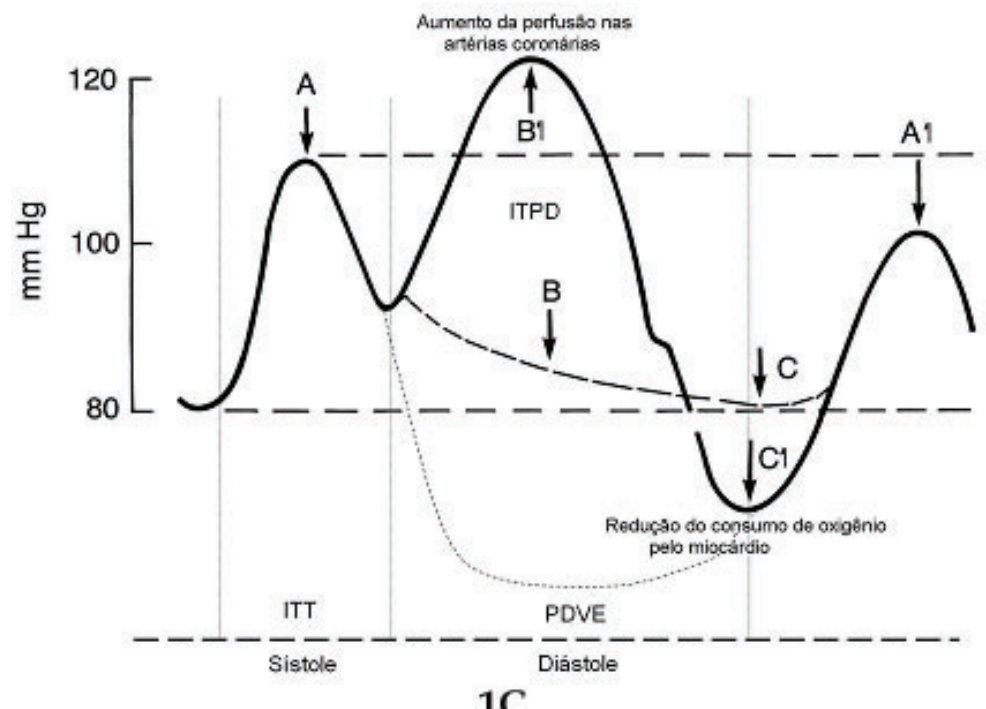

Figura 3. 1A - Balão intra-aórtico posicionado na aorta descendente através da artéria femoral.; 1B - Fases de funcionamento do balão intra-aórtico; 1C - Curva da pressão na aorta ascendente não assistida (ABC) e curva assistida (A1B1C1), onde se nota redução da pressão sistólica com a assistência (de $A$ para $A 1$ ), redução da pressão diastólica final (de $C$ para $C 1$ ) e aumento da pressão durante a diástole (de B para B1). ITT - Índice do Tempo de Tensão, ITPD - Índice do Tempo de Pressão Diastólica e PDVE - Pressão Diastólica do Ventrículo Esquerdo

A desinsuflação deve ter início antes da próxima sístole ventricular. A desinsuflação precoce reduz o tempo de elevação desejável da pressão diastólica e, conseqüentemente, reduz a perfusão coronária. A desinsuflação tardia aumenta a resistência ao desempenho do ventrículo esquerdo pelo aumento da pós-carga. Em situações especiais, como de arritmia incontrolável, hipotensão acentuada ou durante a circulação extracorpórea, o balão pode ser acionado assincronicamente pelo próprio equipamento, neste caso com menor rendimento. $\mathrm{Na}$ fase de desmame da assistência a sincronização do balão pode ser alterada de 1:1(1 do Balão: 1 do Coração), 1:2, a 1:3, permitindo assim que haja acomodação do coração aos valores pressóricos normais.

O balão intra-aórtico é utilizado fundamental- mente na falência do ventrículo esquerdo, pode ser aplicado de forma não usual na disfunção do ventrículo direito posicionando-se o balão no tronco da pulmonar. DARRAH et al., em $1997^{40}$, demonstraram que o emprego do balão em disfunção do ventrículo direito pode melhorar o seu desempenho em até $40 \%$, pela redução da pós-carga imposta pelo ventrículo esquerdo. ARAFA et al., em $2000^{41}$, confirmaram esses achados empregando o balão em disfunção do ventrículo direito após transplante cardíaco.

O princípio do seu funcionamento apóia-se na redução da pós-carga do ventrículo esquerdo e no aumento da pressão de perfusão nas artérias coronárias. Estes benefícios são obtidos de forma mais eficiente com a insuflação sincrônica do balão durante a diástole ventricular e sua desinsuflação durante a sístole. 
A oclusão súbita da aorta descendente pelo balão promove aumento da pressão na porção ascendente da aorta, melhorando a perfusão do coração e do cérebro. Durante a sístole ventricular o balão desinsufla rapidamente facilitando o trabalho ventricular. Este suporte circulatório sincrônico produz: redução do trabalho biventricular, diminuição do consumo de oxigênio pelo miocárdio, redução da pós-carga, auxilia na correção da hipotensão arterial e melhora a perfusão miocárdica. A análise das variáveis hemodinâmicas com o uso do balão demonstra: aumento da pressão arterial, redução da resistência vascular periférica, redução da pressão capilar e aumento do débito cardíaco em até $30 \%$. Estas alterações hemodinâmicas oferecem condições favoráveis para a restauração da função ventricular, podendo ser utilizado também como ponte para o transplante.

As principais contra-indicações à utilização do balão intra-aórtico são:

- Insuficiência da valva aórtica;

- Doença vascular periférica grave;

- Dissecção aguda da aorta.

As principais complicações que podem surgir em virtude do trauma vascular durante a introdução do cateter, ou do uso prolongado, são a introdução do cateter em falsa luz arterial, embolização de placas ateroscleróticas, perfuração ou dissecção da aorta. Durante o funcionamento do balão as principais complicações dizem respeito à ruptura do balão, isquemia do membro inferior, hemólise, sangramentos, trombocitopenia, infecção, trombose arterial ou venosa. O infarto esplênico, a paraplegia e dores abdominais podem ter origem pela oclusão da artéria mesentérica com o uso do balão.

\section{Bombas centrífugas}

Em 1960, Saxton e Andreus ${ }^{42}$ apresentaram os principais estudos sobre a potencialidade das bombas centrífugas, porém, o projeto inicialmente não teve grande sucesso pelo alto índice de hemólise. Em 1966, um esforço combinado entre as Universidades de Minnesota, da Califórnia, de San Diego e da Meditronic $®$ culminou no desenvolvimento de uma bomba do tipo centrífuga ventricular acionada por acoplamento magnético. Em 1978, Golding et al. introduziram o dispositivo na prática clínica e progressivamente ganharam com ampla difusão.

As bombas centrífugas fornecem fluxo contínuo e são acionadas por acoplamento magnético entre o dispositivo descartável que impulsiona o sangue do circuito extracorpóreo e o console. Atualmente existem modelos diferentes no mercado, dispositivos com pás (Sarns-Delphin $囚$ ) e sem pás (Biomedicus $₫ /$ Jostra $\left.{ }^{\circledR}\right)$, sendo que estas últimas apresentam menor efeito hemolítico em relação à primeira.

Este sistema apresenta vantagens sobre os dispositivos de fluxo pulsátil, pois não requerem o uso de válvulas no circuito nem cânulas de maior calibre. As complicações tromboembólicas e hemolíticas, também, são menores quando comparadas com os sistemas de fluxo pulsátil.

O equipamento é constituído por um console, onde se acham dispostos os monitores, controle de rotação de bomba, alarmes e a placa magnética rotatória do console. A base magnética do dispositivo descartável é acoplada à cabeça magnética da bomba. Quando acionada, a bomba promove a rotação dos cones internos do dispositivo e conseqüentemente o sangue que esta no seu interior. Por ação da força centrífuga o sangue é impulsionado para a via de saída do cone, que está conectada ao circuito. $\mathrm{O}$ fluxo sangüíneo é determinado pelo medidor de fluxo posicionado na linha de saída da bomba e depende do número de rotações e da resistência imposta ao circuito.

$\mathrm{Na}$ instalação da assistência ventricular direita, o sangue é drenado em direção à bomba centrífuga por uma cânula posicionada no átrio direito, e, posteriormente, retorna ao paciente por outra cânula introduzida no tronco pulmonar.

$\mathrm{Na}$ assistência esquerda, o sangue é drenado em direção à bomba centrífuga por uma cânula introduzida no átrio esquerdo, e, posteriormente, retorna ao paciente por outra cânula introduzida na aorta ascendente ou na artéria femoral. Na Figura 4 acham-se representadas as diferentes formas de assistência que podem ser empregadas com o auxílio da bomba centrífuga.

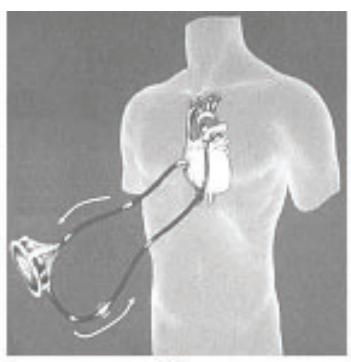

$4 \mathrm{~A}$

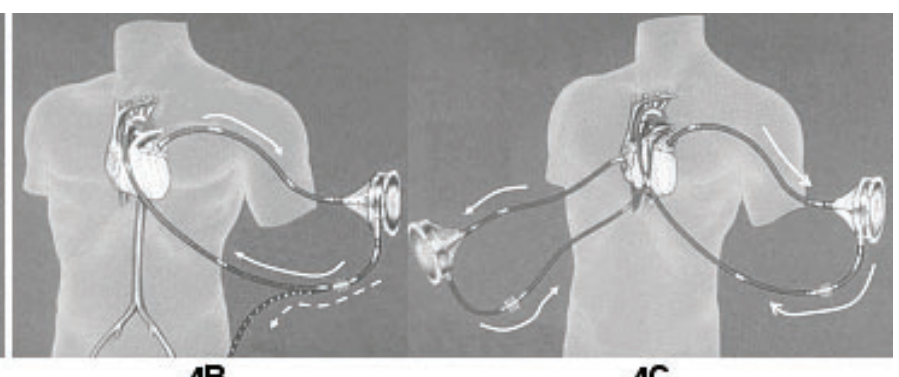

$\mathbf{4 B}$

$4 \mathrm{C}$

Figura 4. A - Assistência Direita; B - Assistência Esquerda e C - Assistência Biventricular 
A anticoagulação é obtida com heparina e a sua monitorização pelo controle seriado do tempo de coagulação ativado (TCA), que deve ser mantido aumentado em $50 \%$ a $100 \%$ dos valores normais. Nos casos onde são mantidos fluxos sangüíneos superiores a 1-2 L/min é possível manter o suporte sem anticoagulação, mesmo por período prolongado. Sem o uso de heparina, contudo, tem-se observado incidência significativa de fenômenos embólicos. Recentemente, tem-se apontado as vantagens do uso de circuitos revestidos por superfície bioativa, denominado de Cormida, que mantém moléculas de heparina fixadas por ligações covalentes, minimizando a ativação do fator contato, ativação de complemento e a trombogênese.

Recentemente, devido à facilidade no manuseio das bombas centrífugas, Phillips et al., têm estendido o uso deste dispositivo como suporte de circulatório em casos de ressuscitação em unidades de emergência, elegendo-se os vasos femorais para acesso das cânulas por punção percutânea. A falência ventricular após cirurgia cardíaca com dificuldade de retirada da circulação extracorpórea tem representado uma das maiores indicações do uso da assistência circulatória centrífuga. Durante a operação cardíaca a instalação deste tipo de assistência torna-se mais simples devido ao fácil acesso às estruturas anatômicas. Nestes casos, prefere-se encaminhar o paciente à unidade de terapia intensiva mantendo-se o tórax fechado apenas com curativo oclusivo. Esta tática evita a compressão do coração, permite melhor controle do sangramento e facilita no momento da retirada do suporte circulatório.

As bombas centrífugas têm sido utilizadas com sucesso nas disfunções do ventrículo direito após o transplante cardíaco em pacientes portadores de pressão pulmonar aumentada e os agentes farmacológicos mostram-se ineficazes. Devido ao pequeno traumatismo produzido nos elementos sangüíneos, este tipo de dispositivo tem sido utilizado em associação com os oxigenadores de membrana em casos de insuficiência respiratória severa como suporte para oxigenação assistida, denominado de ECMO (Extracorporal Membrane Oxygenator).

As principais vantagens com o uso da bomba centrífuga são:

- Menor complexidade e custo em relação aos ventrículos artificiais;

- Simplicidade no manuseio do equipamento;

- Suporte ventrículo direito, esquerdo ou ambos;

- Console pequeno é facilmente transportável;

- Permite monitorização contínua do fluxo sangüíneo;

- Turbulência reduzida, menor traumatismo celular e menor risco de embolia aérea. são:

As principais desvantagens observadas

- Requer monitorização contínua, com perfusionista ao lado do paciente;

- Necessidade de anticoagulação;

- O paciente deve permanecer imobilizado no leito;

- Uso limitado para poucos dias, não superior a 8-10dias;

A interrupção do suporte circulatório deve ser gradual, com redução lenta e programada do fluxo de perfusão e com período de instalação não inferior a 24 horas. A análise evolutiva de comportamento hemodinâmico, ecocardiográfico, metabolismo e as necessidades de drogas vasoativas auxiliam na decisão e na velocidade de desmame da assistência circulatória. A monitorização das curvas de pressão arterial sistêmica e pulmonar, em especial as ondas de ejeção ventricular, orienta na suspensão do suporte circulatório esquerdo e direito, respectivamente.

A sobrevida dos pacientes que receberam suporte circulatório com bomba centrífuga de falência miocárdica após cardiotomia oscila entre $6 \%$ e $26 \%$.

\section{Bombas axiais}

A Hemopump ${ }^{\mathrm{R}}$ foi idealizada por WAMPLER, em 1981, e impulsiona o sangue baseado no período de Arquimedes para construção de bomba d'água. O sistema proposto foi aperfeiçoado, em 1987, por investigadores do Texas Heart Institute. É uma bomba de propulsão sangüínea por rotação de uma pequena turbina, posicionada no interior do ventrículo esquerdo, produzindo um fluxo axial contínuo, em paralelo ao próprio eixo do sistema, sendo utilizado somente em assistência circulatória esquerda.

Este dispositivo aparece como método alternativo de suporte aos já existentes, tendo como características inovadoras a eficiência associada à sua relativa simplicidade de manipulação, podendo ser introduzido por via arterial periférica 
ou pela aorta ascendente. A turbina intra-aórtica gira cerca de 25 mil rotações por minuto e aspira o sangue do ventrículo esquerdo e ejeta-o na aorta, sendo capaz de produzir em débito de 4,7 $\mathrm{L} / \mathrm{min}$ sob regime pressórico de $100 \mathrm{mmHg}$. O sistema dispensa a necessidade de sincronismo e reduz significativamente o trabalho ventricular, por descompressão do ventrículo esquerdo.

A bomba Hemopump teve a sua industrialização interrompida por motivos de quebras mecânicas. Atualmente, está no mercado um sistema que utiliza os mesmos princípios, com vantagens adicionais sobre aquela outra pela sua durabilidade, denominada de Impella ${ }^{\text {}}$.

\section{Ventrículo artificial}

Em 1959, Akuttsu e Kolff ${ }^{44}$ desenvolveram experimentos testando o implante de coração artificial e conseguiram sobrevida dos animais de até uma semana. Cooley em $1969^{45}$ realizou o primeiro implante de coração artificial total, modelo Liotta, em um paciente com disfunção ventricular após cirurgia cardíaca. O suporte circulatório foi mantido por 64 horas e o paciente submetido ao transplante cardíaco.

Em 1978, Cooley et al. ${ }^{46}$ empregaram um dispositivo de assistência ventricular em um paciente de 21 anos que evoluiu com insuficiência miocárdica após dupla trova valvar. Esta assistência serviu de suporte durante cinco dias como ponte, quando foi realizado o transplante.

Após intensas pesquisas foi construído o primeiro coração artificial implantável permanente, denominado de Jarvik-7, sendo implantado por Devries, em 1982, em um paciente que sobreviveu 112 dias. Até 1985, quatro outros pacientes receberam o Jarvik-7, com sobrevida de até 620 dias, porém não isentos de sérias complicações como sangramento e tromboembolismo. Isto fez com que o US Food and Drug Administration (FDA) somente autorizasse o seu implante como ponte para o transplante.

O ventrículo artificial é um dispositivo de propulsão pulsátil que serve para auxiliar o coração como suporte ventricular esquerdo, direito e biventricular. As indicações de implante são semelhantes aos critérios já discutidos nas bombas centrífugas, contudo evita-se sua indicação em pacientes com contra-indicação formal para transplante.

Os modelos pneumáticos, como os Thoratec ${ }^{R}$ e InCor ${ }^{\mathrm{R}}$, são de implante pára-corpóreo e a propulsão sanguínea se dá através da movimentação de uma membrana livre. No modelo Thoratec ${ }^{R}$ a prótese é implantada em posição heterotópica, em paralelo ao ventrículo assistido (direito, esquerdo ou ambos), e apresenta volume sistólico de $65 \mathrm{ml}$. O modelo InCor foi desenvolvido no setor de Bioengenharia do Instituto do Coração do Hospital das Clínicas - FMUSP, e apresenta características básicas de funcionamento semelhante ao Thoratec ${ }^{\mathrm{R}}$, com volume sistólico de $65 \mathrm{ml}$, acionado por uma membrana livre e as válvulas confeccionadas com pericárdio bovino, podendo oferecer fluxo sanguíneo de até $4,81 / \mathrm{min} \cdot \mathrm{m}^{2}$.

O modelo Thermedics ${ }^{R}$ tem sido usado desde 1985. É um dispositivo pneumático totalmente implantável, com fluxo unidirecional que emprega duas válvulas de porco. Tem duas câmaras, a pneumática e a sangüínea, revestidas de titânio e separadas por uma membrana de poliuretana flexível que aciona o fluxo de sangue. O revestimento interior com microesferas de titânio minimiza a formação de coágulos, o que diminuiu a capacidade trombogênica da prótese. O implante é feito em posição intraabdominal, com desvio do sangue do ápice do ventrículo esquerdo e reinfusão na aorta. O console pneumático extracorpóreo aciona o dispositivo, com capacidade para gerar até $8 \mathrm{~L} / \mathrm{min} \cdot \mathrm{m}^{2}$ de débito.

Os ventrículos eletromecânicos, também denominados de "Pusher- Plate", são acionados por uma membrana aderida a uma placa rígida que se movimenta impulsionando o sangue. Os modelos que se destacam são o Novacor ${ }^{R}$ e o TCI heart Mate ${ }^{R}$, de acionamento elétrico e totalmente implantáveis. As baterias que fornecem a energia elétrica podem ser implantadas na cavidade abdominal ou torácica, sendo que os modelos mais recentes são colocados na cintura e acionam o dispositivo mecânico por radiofreqüência. Estes dispositivos podem oferecer até $8 \mathrm{l} / \mathrm{min} \cdot \mathrm{m}^{2}$.

As principais complicações com o uso destes dispositivos são: sangramento, tromboembolismo, hemólise, insuficiência renal, hepática e respiratória. Apresentam como desvantagens a necessidade de toracotomia, associada ou não a laparotomia para seu implante, e o alto custo. As principais vantagens do emprego de ventrículo artificial são:

- Apresentam fluxo pulsátil;

- Permitem suporte prolongado e mobilização do paciente;

- Capacidade de oferecer alto fluxo sanguíneo.

Os principais critérios de contra-indicação 
do uso de ventrículo artificial são:

- Peso inferior a $50 \mathrm{~kg}$ e superfície corpórea inferior a 1,5m2;

- Idade inferior a 65 anos;

- Insuficiência renal ou diálise;

- Infarto pulmonar;

- Hipertensão pulmonar fixa com resistência vascular pulmonar superior a $6 \mathrm{U}$ Wood após uso dos vasodilatadores;

- Insuficiência hepática severa com bilirrubinas superior a $10 \mathrm{mg} \%$ ou biópsia com cirrose;

- Neoplasia não resolvida;

- Coagulopatia;

- Doença cerebrovascular com mal prognóstico;

- Infecção ativa

\section{Coração artificial total}

O coração artificial total é um dispositivo mecânico implantado em posição ortotópica, que substitui integramente o coração nativo, sendo indicado atualmente como ponte para o transplante. Tem se discutido de forma exaustiva sua utilização como substituto definitivo em alguns grupos de pacientes.

O modelo mais difundido é o Jarvik-7, atualmente denominado de Cardio West C-7 $\left(\right.$ Symbion $\left.^{\mathrm{R}}\right)$. Este dispositivo é constituído de duas câmaras pneumáticas confeccionados de poliuretana, que são acopladas aos átrios remanescentes e aos grandes vasos. Cada câmara contém uma válvula de carvão pirolítico, acionados por fontes de energia externa e oferece volume sistólico de $100 \mathrm{ml}$.e débito de 4 a 5L/min, podendo atingir até 10 a 15L/min. Sangramento, tromboembolismo e infecções são as principais complicações.

O modelo Hershey ${ }^{R}$ é um coração artificial total pneumático, onde cada ventrículo é formado por duas câmaras, a sangüínea e a de pressurização, separadas por membrana flexível de poliuretana, com volume sistólico de $70 \mathrm{ml}$, sendo implantado em posição heterotópica. A experiência clínica brasileira é inexistente com dispositivos de coração artificial total.

Tem crescido o interesse por dispositivos não pulsáteis nos últimos anos, em 1989 surge o The Jarvik 2000 LVAD, Figura 5. Inicialmente utilizado apenas em pacientes como ponte para transplante nos EUA, atualmente na Europa vem sendo utilizado como terapia definitiva. Em 1991 surge o modelo da MicroMed De-Bakey assist device, apresentado na Figura 5.

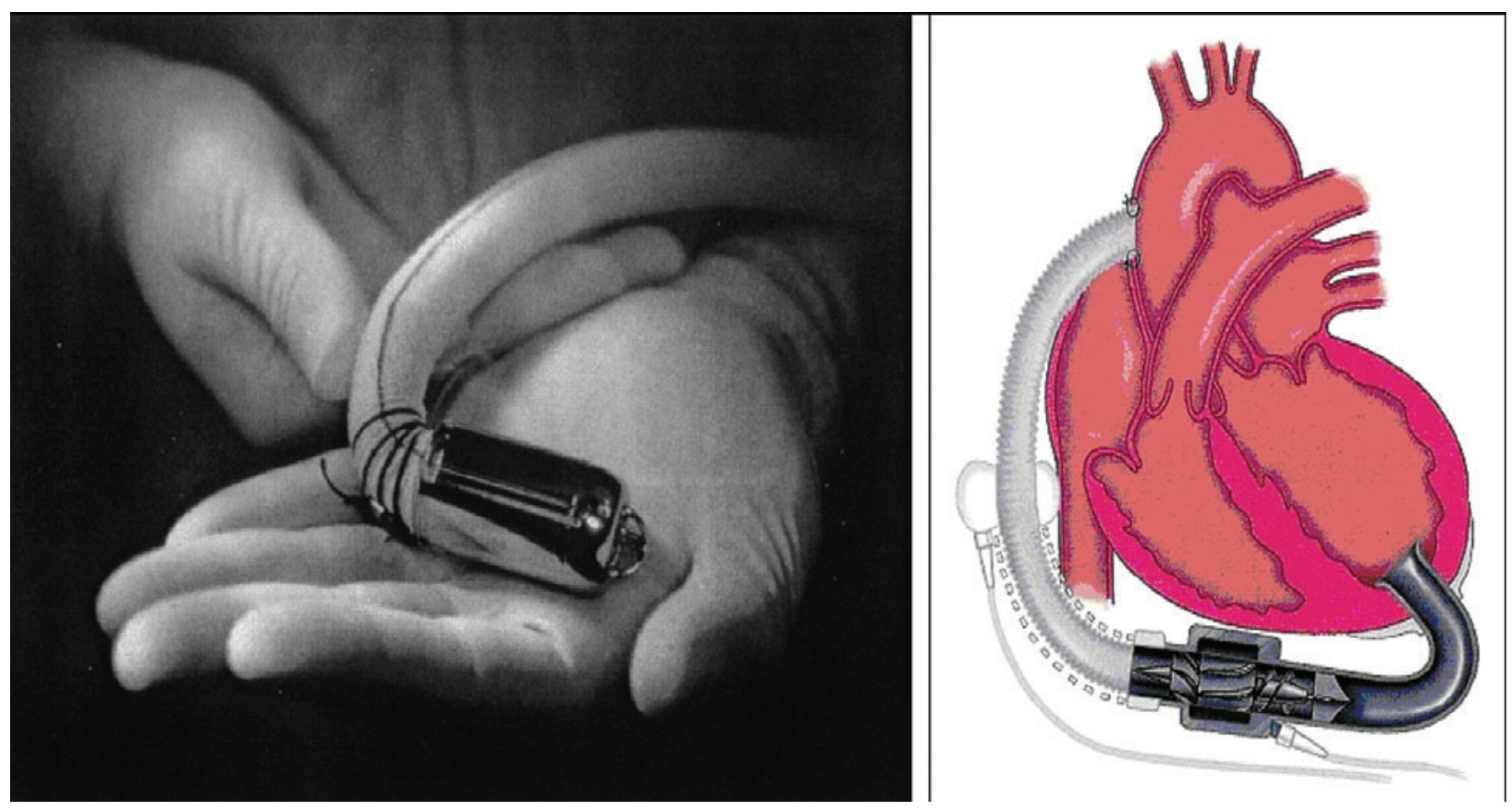

Figura 5. Assistência Circulatória Mecânica com o modelo da MicroMed De-Bakey assist device 
Fiorelli Al, Oliveira Junior JL, Coelho GHB, Rocha DC. Mechanical circulatory support: why and when. Rev Med (São Paulo). 2008 jan.-mar.;87(1):1-15.

\begin{abstract}
Heart failure has advanced throughout last years due to the aging of population, the higher survival rate after acute myocardial infarction due to trombolitics use, the advance in heart surgeries and the improvement in cardiomyopathies diagnosis. The disease has no cure and its natural course is unavoidable, despite different attempts to change it. The best until now is retarding its outcome. Heart transplant currently represents the only way to drastically change this scenery, by offering significative improvement in quality of life and in long-term survival. However, the procedure is limited to few people, mainly because of the scantiness of organ donors. Many patients die waiting for the surgery. Mechanical circulatory support arises as an artificial support that allows many to reach the transplant, working as bridge to the procedure. In acute cases of heart failure, in which the myocardium shows recovery conditions, the mechanical support allows offering propitious hemodynamic conditions for the patient not to develop multiple organ failure and for the myocardium in relative rest to have recovery conditions. This last form is called bridge to recovery. Recently, the use of artificial ventricles and hearts as therapy, such as definitive implant in those patients who have counterindication to the transplant or refuse the procedure, is under discussion. The aim of the present article is to show a wide review about the roll that the mechanical circulatory support plays as an assistant method in the treatment of heart failure.
\end{abstract}

KEY WORDS: Heart failure congestive/pathology. Assisted circulation/methods. Heart transplantation/trends. Risk factors. Quality of life.

\section{REFERÊNCIAS}

1. Chen FY, Cohn LH. The surgical treatment of heart failure. A new frontier: nontransplant surgical alternatives in heart failure. Cardiol. Rev. 2002;10(6):326-33.

2. Birks EJ, Tansley PD, Hardy JBS, George RS, Bowles $\mathrm{CT}$, Path MB, et al. Left ventricular assist device and drug theraphy for the reversal of heart failure. $\mathrm{N} \mathrm{Engl}$ J Med. 2006;355:1873-84.

3. Pfeffer MA, Braunwald E. Ventricular remodeling after myocardial infarction: experimental observations and clinical implications. Circulation.1990;81:1161-72.

4. Swynghedauw B. Molecular mechanisms of myocardial remodeling. Physiol Rev. 1999;79:215-62.

5. Lowes BD, Gilbert EM, Abraham WT, Minobe WA, Larrabee $\mathrm{P}$, Ferguson D, et al. Myocardial gene expression in di-lated cardiomyopathy treated with betablocking agents. N Engl J Med. 2002;346:135765.

6. Iwanaga $Y$, Hoshijima M, Gu Y, Iwatate M, Dieterle $\mathrm{T}$, Ikeda $\mathrm{Y}$, et al. Chronic phospholamban inhibition prevents progressive cardiac dysfunction and pathological remodeling after infarction in rats. J Clin Invest. 2004;113:727-36.

7. Yacoub MH. A novel strategy to maximize the efficacy of left ventricular assist devices as a bridge to recovery. Eur Heart J. 2001;22:534-40.

8. Starling RC. Inducible nitric oxide synthase in severe human heart failure: impact of mechanical unloading. J Am Coll Cardiol. 2005;45:1425-7.

9. Pieske B. Reverse remodelling in heart failure - fact or fiction? Eur Heart J Suppl. 2004;6:D66-D78.

10. Zafeiridis A, Jeevanandam V, Houser SR, Margulies KB. Regression of cellular hypertrophy after left ventricular assist device support. Circulation. 1998;98:656-62.

11. Dipla K, Mattiello JA, Jeevanandam V, Houser SR, Margulies KB. Myocyte recovery after mechanical circulatory support in humans with end-stage heart failure. Circulation. 1998;97:2316-22.

12. Terracciano CMN, Harding SE, Adamson D, Koban M, Tansley P, Birks EJ, et al. Changes in sarcolemmal $\mathrm{Ca}$ entry and sarcoplasmic reticulum $\mathrm{Ca}$ content in ventricular myocytes from patients with end-stage heart failure following myocardial recovery after combined pharmacological and ventricular assist device therapy. Eur Heart J. 2003;24:1329-39.

13. Terracciano CMN, Hardy J, Birks EJ, Khaghani A, Banner NR, Yacoub MH. Clinical recovery from endstage heart failure using left ventricular assist device and pharmacologic therapy correlates with increased sarcoplasmic reticulum calcium content, but not with regression of cellular hypertrophy. Circulation. 2004;109:2263-5.

14. United Network for Organ Sharing (UNOS). Annual report of the U.S Scientific Registry for organ transplantation and the organ procurement and transplantation network. US Department of Health and Human Services; 1999.

15. Turer AT, Rao SV. Device therapy in the management of congestive heart failure. Cardiol Rev. 2005;13:130-8.

16. Jaski BE, Kim J, Maly RS, Branch KR, Adamson $R$, Favrot LK, et al. Effects of exercise during longterm support with a left ventricular assist device: results of the experience with left ventricular assist device with exercise (EVADE) pilot trial. Circulation. 1997;95:2401-6.

17. Mancini D, Goldsmith R, Levin H, Beniaminovitz A, Rose $\mathrm{E}$, Catanese $\mathrm{K}$, et al. Comparison of exercise performance in patients with chronic severe heart failure versus left ventricular assist devices. Circulation. 1998;98:1178-83.

18. Jaski BE, Lingle RJ, Kim J, Branch KR, Goldsmith R, Johnson MR, et al. Comparison of functional capacity in patients with end-stage heart failure following 
implantation of a left ventricular assist device versus heart transplantation: results of the experience with left ventricular assist device with exercise trial. J Heart Lung Transplant. 1999;18:1031-40.

19. Hetzer R, Muller J, Weng Y, Wallukat G, Spiegelsberger $\mathrm{S}$, Loebe M. Cardiac recovery in dilated cardiomyopathy by unloading with a left ventricular assist device. Ann Thorac Surg. 1999;68:742-9.

20. Madigan JD, Barbone A, Choudhri AF, Morales $\mathrm{DL}$, Cai $\mathrm{B}, \mathrm{Oz} M C$, et al. Time course of reverse remodeling of the left ventricle during support with a left ventricular assist device. J Thorac Cardiovasc Surg. 2001;121:902-8.

21. Westaby $S$, Jin $X Y$, Katsumata $T$, Taggart DP, Coats AJ, Frazier $\mathrm{OH}$. Mechanical support in dilated cardiomyopathy: signs of early left ventricular recovery. Ann Thorac Surg. 1997;64:1303-8.

22. Torre-Amione G, Stetson SJ, Youker KA, Durand JB, Radovanceirc B, Delgado RM, et al. Decreased expression of tumor necrosis factor-alpha in failing human myocardium after mechanical circulatory support: a potential mechanism for cardiac recovery. Circulation. 1999;100:1189-93.

23. Rose EA, Gelijns AC, Moskowitz AJ, Heitjan DF, Stevenson LW, Dembitsky JH et al. Long-term mechanical left ventricular assistance for end-stage heart failure. N Engl J Med. 2001;345:1435-43.

24. Young JB. Healing the heart with ventricular assist device therapy: mechanisms of cardiac recovery. Ann Thorac Surg. 2001;71(3 Suppl):S210-9.

25. Felker GM, Rogers JG. Same bridge, new destinations rethinking paradigms for mechanical cardiac support in hearth failure. J Am Coll Cardiol. 2006;47(5):930-2.

26. Paul B, BolnoJ, Yasha K. Physiologic and hemodynamic basis of ventricular assist devices. Cardiol Clin. 2003;21:15-27.

27. Bruckner BA, Stetson SJ, Perez-Verdia A, Youker $\mathrm{KA}$, Radovancevic B, Connelly $\mathrm{JH}$, et al. Regression of fibrosis and hypertrophy in failing myocardium following mechanical circulatory support. J Heart Lung Transplant. 2001;20:457-64.

28. Vatta M, Stetson SJ, Perez-Verdia A, Entaman ML, Noon GP, Torre-Amione G, et al. Molecular remodeling of dystrophin in patients with end-stage cardiomyopathies and reversal in patients on assistance-device therapy. Lancet. 2002;359:936-41.

29. Petrofski JA, Hoopes CW, Bashore TM, Russell SD, Milano CA. Mechaniocal ventricular support lowers pulmonary vascular resistance in a patient with congential heart disease. Ann Thorac Surg. 2003:75:1005-7.

30. Young JB, Rogers JG, Portner PM, et al. Bridge to eligibility: LVAS support in patients with relative contraindications to transplantation. Heart Lung Transplant. 2005;24:S75.

31. Fraizer OH, Myers TJ, Gregoric ID, Than T, Delgado $\mathrm{R}$, Coitoru M, et al. Initial clinical experience with the Jarvik 2000 implantable axial-flow left ventricular assist system. Circulation. 2002;105:2855-60.

32. Mancini DM, Beniaminovitz A, Levin $\mathrm{H}$, Catanese $\mathrm{K}$, Flannery M, DiTullio $\mathrm{M}$, et al. Low incidence of myocardial recovery after left ventricular assist device implantation in patients with chronic heart failure. Circulation. 1998;98:2383-9.

33. Barbone A, Holmes JW, Heerdt PM, The AH, Naka Y, Joshi N, et al. Comparison of right and left ventricular responses to left ventricular assist device support in patients with severe heart failure: a primare role of mechanical unloading underlying reverse remodeling. Circulation. 2001;104:670-5.

34. Altemose GT, Gritsus V, Jeevanandam V, Goldman B, Margulies KB. Altered myocardial phenotype after mechanical support in human beings with advanced cardiomyopathy. J Heart Lung Transplant. 1997;16:765-73.

35. Brucker BA, Stetson SJ, Farmer JA, Radovancevic $\mathrm{B}$, Frazier $\mathrm{OH}$, Noon GP, et al. The implications for cardiac recovery of left ventricular assist device support on myocardial collagen content. Am J Surg. 2000;180:498-502.

36. Marshall I Hertz, Paul Aurora, Mark M Boucek, Jason D Christie, Fabienne Dobbels, Leah B Edwards, Berkeley M Keck, Axel O Rahmel, Amanda W Rowe, David O Taylor, Elbert P Trulock. Registry of the International Society for Heart and Lung Transplantation: introduction to the 2007 annual reports-100,000 transplants and going strong. J Heart Lung Transplant. 2007;26(8):763-8.

37. Harken DE. Presentation at the International College of Cardiology, Brussels, Belgium; 1958.

38. Moulopoulos SD, Topaz S, Hiller K, Kolff WJ. Proper pressure curve shape in ventricles of intrathoracic mechanical hearts. J Appl Physiol. 1962;17:1025-8.

39. Kantrowitz A, Tjonneland S, Freed PS, Phillips SJ. Initial clinical experience with intraaortic balloon pumping in cardiogenic shock. JAMA. 1968;203(2):113-8.

40. William CD, Sharpe MD, Gerard MG, Andy Neal AHT. Intraaortic balloon counterpulsation improves right ventricular failure resulting from pressure overload. Ann Thorac Surg. 1997;64:1718-23.

41. Osama E, Arafa OR, Geiran KA, Erik F, Svein S, Jan LS. Intraaortic balloon pumping for predominantly right ventricular failure after heart transplantation. Ann Thorac Surg. 2000;70:1587-93.

42. Saxton GA Jr, Andrews CB. An ideal heart pump with hydrodynamic characteristics analogous to the mammalian heart. Trans Am Soc Artif Intern Organs. 1960;6:288-91.

43. Golding LR, Harasaki H, Loop FD, Sukalac R, Reich $\mathrm{S}$, Nosé $\mathrm{Y}$. Use of a centrifugal pump for temporary left ventricular assist system. Trans Am Soc Artif Intern Organs. 1978;24:93-7.

44. Akutsu T, Dreyer B, Kolff WJ. Polyurethane artificial heart valves in animals. J Appl Physiol. 1959; $14: 1045-8$.

45. Cooley DA, Liotta D, Hallman GL, Bloodwell RD, Leachman RD, Milam JD. Orthotopic cardiac prosthesis for two-staged cardiac replacement. Am J Cardiol. 1969;24(5):723-30.

46. Partial artificial heart (ALVAD) use with subsequent cardiac and renal allografting in a patient with stone heart syndrome. Artif Organs. 1978;2(4):413-20. 